\title{
STABILITY ANALYSIS OF THE SEQUENTIAL PARTIAL UPDATE LMS ALGORITHM
}

\author{
Mahesh Godavarti and Alfred O. Hero III \\ Department of Electrical Engineering \& Computer Science \\ The University of Michigan \\ Ann Arbor, MI 48105 \\ mgodavar@eecs.umich.edu, hero@eecs.umich.edu
}

\begin{abstract}
Partial updating of LMS filter coefficients is an effective method for reducing the computational load and the power consumption in adaptive filter implementations. The Sequential Partial Update LMS algorithm is one popular algorithm in this category. In [5] a first order stability analysis of this algorithm was performed on wide sense stationary signals under the restrictive assumption of small step size parameter $\mu$. The necessary and sufficient condition derived on $\mu$ for convergence in the mean was identical to the one for guaranteeing stability in the mean of LMS. In [7] first order sufficient conditions were derived for stability without the aforementioned small $\mu$ assumption. The sufficient region of convergence derived was smaller than that of regular LMS. In this paper, we establish that for stationary signals the sequential algorithm converges in mean for the same values of the step size parameter $\mu$ for which the regular LMS does. In other words, we show that the conclusion drawn in [5] holds without the restrictive assumption of small $\mu$. We also derive sufficient conditions for stability on $\mu$ for cyclo-stationary signals.
\end{abstract}

\section{INTRODUCTION}

Partial updating of the LMS adaptive filter has been proposed to reduce computational costs and power consumption $[2,3,4]$ which is quite attractive in the area of of mobile computing and communications. Partial update algorithms have application in many fields including adaptive beamforming, channel equalization in communications and space-time modulation/coding. Sequential Partial Update LMS algorithm is one such algorithm. However, for this algorithm theoretical performance predictions on convergence rate and steady state tracking error are more difficult to derive than for standard full update LMS. Accurate theoretical predictions are important as it has been observed that for the non-stationary signal scenario the standard LMS conditions on the step size parameter fail to ensure convergence of S-LMS.

In [5], conditions were derived under the assumption of small step-size parameter $(\mu)$ which turned out to be the same as those for the standard LMS algorithm. We were interested in investigating the Sequential Partial Update LMS Algorithm (S-LMS) under large $\mu$ conditions for which faster convergence rates could be

This work was supported by a Graduate Fellowship to the first author from the Department of Electrical Engineering and Computer Science at the University of Michigan and by the Department of Defense Research \& Engineering (DDR\&E) Multidisciplinary University Research Initiative (MURI) on "Low Energy Electronics Design for Mobile Platforms" managed by the Army Research Office (ARO) under ARO grant DAAH04-96-1-0337. Corresponding author: Mahesh Godavarti, mgodavar@eecs.umich.edu, (734)-764-4321 (voice), (734)-763-8041 (fax) achieved. In [7], we derived a less restrictive sufficient condition on $\mu$ for stationary signals. However, we were unable to find an example of a stationary signal for which regular LMS was stable but S-LMS unstable. We also point out that the motivating example in Section 2 of [7] is incorrect.

This has led us to look for more accurate bounds on $\mu$ which hold for stationary signals and arbitrary fixed sequence of partial updates. Here, we prove conclusively that for stationary signals first order stability of LMS implies first order stability of S-LMS without any assumptions on $\mu$ (Theorem 2 ). We also extend the analysis in [7] to cyclo-stationary signals. We show that for the cyclo-stationary case there exists some $\mu$ such that use of it in SLMS could lead to divergence even if full update LMS converges for this $\mu$.

The organization of the paper is as follows. First in Section 2, a brief description of the sequential partial update algorithm is given. The algorithm with arbitrary sequence of updates is analyzed for the case of stationary signals in Section 3 . This is followed by the analysis of algorithm with the special case of alternate even and odd coefficient updates for cyclo-stationary signals in Section 4 . In Section 5 an example is given to illustrate the usefulness of the bounds on step-size derived in Section 4. Finally, conclusions and directions for future work are indicated in Section 6.

\section{ALGORITHM DESCRIPTION}

The block diagram of S-LMS for a $N$-tap LMS filter with alternating even and odd coefficient updates is shown in Figure 1

It is assumed that the LMS filter is a standard FIR filter of even length, $N$. For convenience, we start with some definitions. Let $\left\{x_{k}\right\}$ be the input sequence and let $\left\{w_{i, k}\right\}$ denote the coefficients of the adaptive filter. Define

$$
\begin{aligned}
W_{k} & =\left[\begin{array}{llll}
w_{1, k} & w_{2, k} & \ldots & w_{N, k}
\end{array}\right]^{T} \\
X_{k} & =\left[\begin{array}{lllll}
x_{k} & x_{k-1} & x_{k-2} & \ldots & x_{k-N+1}
\end{array}\right]^{T}
\end{aligned}
$$

where the terms defined above are for the instant $k$. In addition, Let $d_{k}$ denote the desired response. In typical applications $d_{k}$ is a known training signal which is transmitted over a noisy channel with unknown FIR transfer function.

In this paper we assume that $d_{k}$ itself obeys an FIR model given by $d_{k}=W_{o p t}^{H} X_{k}+n_{k}$ where $W_{o p t}$ are the coefficients of an FIR model given by $W_{\text {opt }}=\left[w_{1, o p t} \ldots w_{N, \text { opt }}\right]^{T}$. Here $\left\{n_{k}\right\}$ is assumed to be a zero mean i.i.d sequence that is independent of the input sequence $\left\{x_{k}\right\}$.

For description purposes we will assume that the filter coefficients can be divided into $P$ mutually exclusive subsets of equal size, i.e. the filter length $N$ is a multiple of $P$. For convenience, 
define the index set $S=\{1,2, \ldots, N\}$. Partition $S$ into $P$ mutually exclusive subsets of equal size, $S_{1}, S_{2}, \ldots, S_{P}$. Define $I_{i}$ by zeroing out the $j^{t h}$ row of the identity matrix $I$ if $j \notin S_{i}$. In that case, $I_{i} X_{k}$ will have precisely $\frac{N}{P}$ non-zero entries. Let the sentence "choosing $S_{i}$ at iteration $k$ " stand to mean "choosing the weights with their indices in $S_{i}$ for update at iteration $k$ ".

The S-LMS algorithm is described as follows. At a given iteration, $\mathrm{k}$, one of the sets $S_{i}, i=1, \ldots, P$, is chosen in a predetermined fashion and the update is performed.

$$
w_{k+1, j}= \begin{cases}w_{k, j}+\mu e_{k}^{*} x_{k, j} & \text { if } j \in S_{i} \\ w_{k, j} & \text { otherwise }\end{cases}
$$

where $e_{k}=d_{k}-W_{k}^{H} X_{k}$. The above update equation can be written in a more compact form in the following manner

$$
W_{k+1}=W_{k}+\mu e_{k}^{*} I_{i} X_{k}
$$

In the special case of even and odd updates, $P=2$ and $S_{1}$ consists of all even indices and $S_{2}$ of all odd indices as shown in Figure 1.

We also define the coefficient error vector as

$$
V_{k}=W_{k}-W_{o p t}
$$

which leads to the following coefficient error vector update for SLMS when $k$ is odd

$$
\begin{aligned}
V_{k+2}= & \left(I-\mu I_{2} X_{k+1} X_{k+1}^{H}\right)\left(I-\mu I_{1} X_{k} X_{k}^{H}\right) V_{k}+ \\
& \mu\left(I-\mu I_{2} X_{k+1} X_{k+1}^{H}\right) n_{k} I_{1} X_{k}+\mu n_{k+1} I_{2} X_{k+1}
\end{aligned}
$$

and the following when $k$ is even

$$
\begin{aligned}
V_{k+2}= & \left(I-\mu I_{1} X_{k+1} X_{k+1}^{H}\right)\left(I-\mu I_{2} X_{k} X_{k}^{H}\right) V_{k}+ \\
& \mu\left(I-\mu I_{1} X_{k+1} X_{k+1}^{H}\right) n_{k} I_{2} X_{k}+\mu n_{k+1} I_{1} X_{k+1}
\end{aligned}
$$

\section{ANALYSIS: STATIONARY SIGNALS}

Assuming that $\left\{x_{k}\right\}$ is a WSS random sequence, we analyze the convergence of the mean coefficient error vector $E\left[V_{k}\right]$. We make the standard assumptions that $V_{k}$ and $X_{k}$ are mutually uncorrelated and that $X_{k}$ is independent of $X_{k-1}$ [1]. These assumptions are somewhat restrictive but greatly simplify the analysis. For regular full update LMS algorithm the recursion for $E\left[V_{k}\right]$ is given by

$$
E\left[V_{k+1}\right]=(I-\mu R) E\left[V_{k}\right]
$$

where $I$ is the $N$-dimensional identity matrix and $R=E\left[X_{k} X_{k}^{H}\right]$ is the input signal correlation matrix. The necessary and sufficient condition for stability of the recursion is given by

$$
0<\mu<2 / \lambda_{\max }
$$

where $\lambda_{\max }$ is the maximum eigen-value of the input signal correlation matrix $R$.

Taking expectations under the same assumptions as above, using the independence assumption on the sequences $X_{k}, n_{k}$, the mutual independence assumption on $X_{k}$ and $V_{k}$, and simplifying we obtain for odd $k$ when S-LMS is operating under the special case of alternate even and odd updates

$$
E\left[V_{k+2}\right]=\left(I-\mu I_{2} R\right)\left(I-\mu I_{1} R\right) E\left[V_{k}\right]
$$

and for even $k$

$$
E\left[V_{k+2}\right]=\left(I-\mu I_{1} R\right)\left(I-\mu I_{2} R\right) E\left[V_{k}\right]
$$

It can be shown that under the above assumptions on $X_{k}, V_{k}$ and $d_{k}$, the convergence conditions for even and odd update equations are identical. We therefore focus on (7). Now to ensure stability of (8), the eigenvalues of $\left(I-\mu I_{2} R\right)\left(I-\mu I_{1} R\right)$ should lie inside the unit circle. We will show that if the eigenvalues of $I-\mu R$ lie inside the unit circle then so do the eigenvalues of $\left(I-\mu I_{2} R\right)\left(I-\mu I_{1} R\right)$.

Now, if instead of just two partitions of even and odd coeffcients $(P=2)$ we have any number of arbitrary partitions $(P \geq 2)$ then the update equations can be similarly written as above with $P>2$. Namely,

$$
E\left[V_{k+P}\right]=\prod_{i=1}^{P}\left(I-\mu I_{i} R\right) E\left[V_{k}\right]
$$

We will show that for any arbitrary partition of any size $(P \geq$ 2); S-LMS converges in the mean if LMS converges in the mean(Theorem 2). The case $P=2$ follows as a special case.

We will show that if $R$ is a positive definite matrix of dimension $N \times N$ with eigenvalues lying in the open interval $(0,2)$ then $\prod_{i=1}^{P}\left(I-I_{i} R\right)$ has eigenvalues inside the unit circle. $I_{i}$, $i=1, \ldots, P$ is obtained from $I$, the identity matrix of dimension $N \times N$, by zeroing out some rows in $I$ such that $\sum_{i=1}^{M} I_{i}$ is positive definite.

The following theorem is used in proving the main result in Theorem 2.

Theorem 1 [6, Prob. 16, page 410] Let $B$ be an arbitrary $N \times N$ matrix. Then $\rho(B)<1$ if and only if there exists some positive definite $N \times N$ matrix $A$ such that $A-B^{H} A B$ is positive definite. $\rho(B)$ denotes the spectral radius of $B(\rho(B)=$ $\left.\max _{1, \ldots, N}\left|\lambda_{i}(B)\right|\right)$

Theorem 2 Let $R$ be a positive definite matrix of dimension $N \times$ $N$ with $\rho(R)=\lambda_{\max }(R)<2$ then $\rho\left(\prod_{i=1}^{P}\left(I-I_{i} R\right)\right)<1$ where $I_{i}, i=1, \ldots, P$ are obtained by zeroing out some rows in the identity matrix $I$ such that $\sum_{i=1}^{P} I_{i}$ is positive definite. Thus $S$-LMS converges in the mean if LMS converges in the mean.

Proof: Let $\mathbf{x}_{0} \in \mathbb{C}^{N}$ be an arbitrary non-zero vector of length $N$. Let $\mathbf{x}_{i}=\left(I-I_{i} R\right) \mathbf{x}_{i-1}$. Also, let $\mathbf{P}=\prod_{i=1}^{P}\left(I-I_{i} R\right)$.

First we will show that $\mathbf{x}_{i}^{H} R \mathbf{x}_{i} \leq \mathbf{x}_{i-1}^{H} R \mathbf{x}_{i-1}-\alpha \mathbf{x}_{i-1}^{H} R I_{i} R \mathbf{x}_{i-1}$, where $\alpha=\frac{1}{2}\left(2-\lambda_{\max }(R)\right)>0$.

$$
\begin{aligned}
\mathbf{x}_{i}^{H} R \mathbf{x}_{i}= & \mathbf{x}_{i-1}^{H}\left(I-R I_{i}\right) R\left(I-I_{i} R\right) \mathbf{x}_{i-1} \\
= & \mathbf{x}_{i-1}^{H} R \mathbf{x}_{i-1}-\alpha \mathbf{x}_{i-1}^{H} R I_{i} R \mathbf{x}_{i-1}- \\
& \beta \mathbf{x}_{i-1}^{H} R I_{i} R \mathbf{x}_{i-1}+\mathbf{x}_{i-1}^{H} R I_{i} R I_{i} R \mathbf{x}_{i-1}
\end{aligned}
$$

where $\beta=2-\alpha$. If we can show $\beta R I_{i} R-R I_{i} R I_{i} R$ is positive semi-definite then we are done. Now

$$
\beta R I_{i} R-R I_{i} R I_{i} R=\beta R I_{i}\left(I-\frac{1}{\beta} R\right) I_{i} R
$$

Since $\beta=\left(1+\lambda_{\max }(R) / 2\right)>\lambda_{\max }(R)$ it is easy to see that $I-\frac{1}{\beta} R$ is positive definite. Therefore, $\beta R I_{1} R-R I_{1} R I_{1} R$ is positive semi-definite and

$$
\mathbf{x}_{i}^{H} R \mathbf{x}_{i} \leq \mathbf{x}_{i-1}^{H} R \mathbf{x}_{i-1}-\alpha \mathbf{x}_{i-1}^{H} R I_{i} R \mathbf{x}_{i-1}
$$




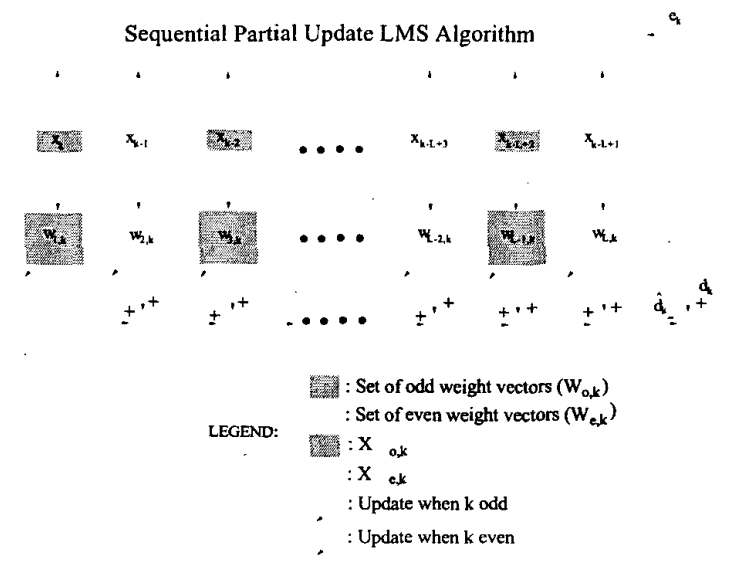

Fig. 1. Block diagram of S-LMS for the special case of alternating grentodd coefficient ypdate

Hig. 2. Trajectory of $w_{1}, k$ and $w_{2, k}$ for $\mu=0.33$ for a 2-tap adaptive filter in a cyclo-stationary signal environment described

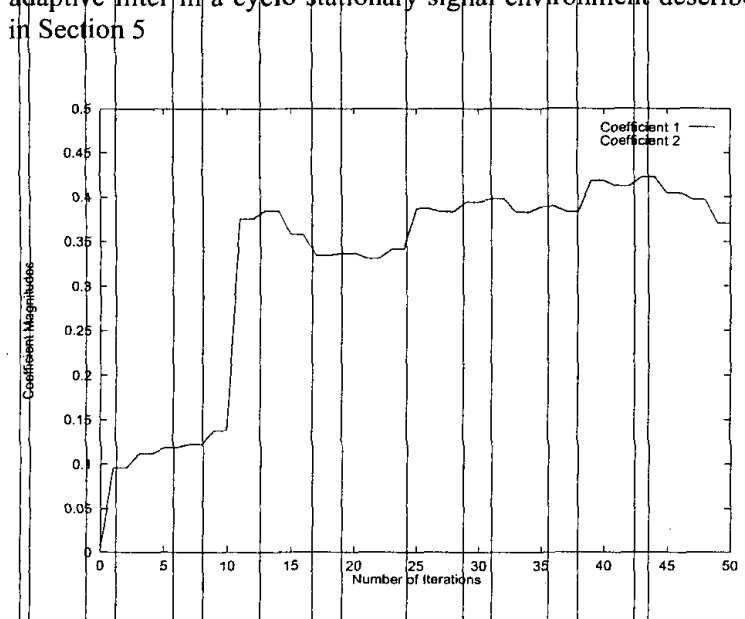

Hig. 3. Trajectory of $w_{1, k}$ and $\left.w_{2}\right|_{k}$ for $\mu=0.0254$ forr the same adaptive system as shown in Figure 2

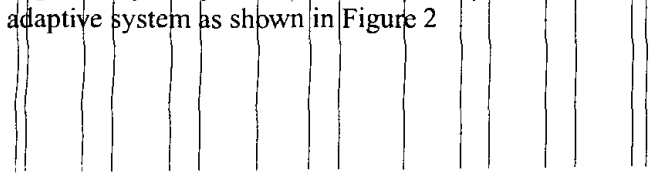

\section{CONCLUSION}

We have analyzed the alternating odd/even partial update LMS algorithm and we have derived stability bounds on step-size parameter $\mu$ for wide sense stationary and cyclo-stationary signals based on extremal properties of the matrix 2-norm. For the case of wide sense stationary signals we have shown that if the regular LMS algorithm converges in mean then so does the sequential LMS algorithm for the general case of arbitrary but fixed ordering of the sequence of partial coefficient updates. For cyclo-stationary signals the bounds derived may not be the weakest possible bounds but they do provide the user with a useful sufficient condition on $\mu$ which ensures convergence in the mean. We believe the analysis undertaken in this paper is the first step towards deriving concrete bounds on step-size without making small $\mu$ assumptions. The analysis also leads directly to an estimate of mean convergence rate.

In the future, it would be useful to analyze partial update algorithm, without the assumption of independent snapshots and also, if possible, perform a second order analysis (mean square convergence). Furthermore, asS-LMS exhibits poor convergence in non-stationary signal scenarios [8] it is of interest to develop new partial update algorithms with better convergence properties. One such algorithm based on randomized partial updating of filter coefficients is described in [8].

\section{REFERENCES}

[1] S. T. Alexander, Adaptive signal processing, SpringerVerlag, New York NY, 1986.

[2] M. J. Gingell, B. G. Hay, and L. D. Humphrey, "A Block Mode Update Echo Canceller Using Custom LSI," GLOBECOM Conference Record, Vol. 3, pp. 1394-1397, Nov. 1983.

[3] M. Godavarti, "Implementation of a G.165 Line Echo Canceller on Texas Instruments' TMS320C3x and TMS320C54x chips," ICSPAT Conference Proceedings, pp. 65-69, Sep. 1997.

[4] D. Messerschmitt, D. Hedberg, C. Cole, A. Haoui and P. Winship, "Digital Voice Echo Canceller with a TMS32020," Application report: SPRA129, Texas Instruments.

[5] S. C. Douglas, "Adaptive Filers Employing Partial Updates," IEEE Trans. Circuits and Systems-II: Analog and Digital Signal Processing, vol. 44, pp. 209-216, March 1997.

[6] R. A. Horn and C. R. Johnson, Matrix Analysis, Cambridge University Press, 1996.

[7] M. Godavarti, A. O. Hero, "Stability Bounds on Step-Size for the Partial Update LMS Algorithm," Proc. International Conference on Acoustic, Speech and Signal Processing, Phoenix, AZ, pp. 1677-1680, Mar. 1999.

[8] M. Godavarti, A. O. Hero, "Stochastic Partial Update LMS Algorithm for Adaptive Arrays," IEEE Sensor Array and Multichannel Signal Processing Workshop, Cambridge, MA, Mar. 2000. 
Combining the above inequality for $i=1, \ldots, P$, we note that $\mathbf{x}_{P}^{H} R \mathbf{x}_{P}<\mathbf{x}_{0}^{H} R \mathbf{x}_{0}$ if $\mathbf{x}_{i-1}^{H} R I_{i} R \mathbf{x}_{i-1}>0$ for at least one $i, i=1, \ldots, P$. We will show by contradiction that is indeed the case.

Suppose not, then $\mathrm{x}_{i-1}^{H} R I_{i} R \mathrm{x}_{i-1}=0$ for all $i, i=1, \ldots, P$. Since, $\mathbf{x}_{0}^{H} R I_{1} R \mathbf{x}_{0}=0$ this implies $I_{1} R \mathbf{x}_{0}=0$. Therefore, $\mathbf{x}_{1}=$ $\left(I-I_{1} R\right) \mathrm{x}_{0}=\mathrm{x}_{0}$. Similarly, $\mathbf{x}_{i}=\mathrm{x}_{0}$ for all $i, i=1, \ldots, P$. This in turn implies that $\mathbf{x}_{0}^{H} R I_{i} R \mathbf{x}_{0}=0$ for all $i, i=1, \ldots, P$ which is a contradiction since $R\left(\sum_{i=1}^{P} I_{i}\right) R$ is a positive-definite matrix and $0=\sum_{i=1}^{P} \mathbf{x}_{0}^{H} R I_{i} R \mathbf{x}_{0}=\mathbf{x}_{0}^{H} R\left(\sum_{i=1}^{P} I_{i}\right) R \mathbf{x}_{0} \neq 0$.

Finally, we conclude that

$$
\begin{aligned}
\mathbf{x}_{0}^{H} \mathbf{P}^{H} R \mathbf{P} \mathbf{x}_{0} & =\mathbf{x}_{P}^{H} R \mathbf{x}_{P} \\
& <\mathbf{x}_{0}^{H} R \mathbf{x}_{0}
\end{aligned}
$$

Since $\mathbf{x}_{0}$ is arbitrary we have $R-\mathbf{P}^{H} R \mathbf{P}$ to be positive definite so that applying Theorem 1 we conclude that $\rho(\mathbf{P})<1$.

Finally, if LMS converges in the mean we have $\rho(I-\mu R)<1$ or $\lambda_{\max }(\mu R)<2$. Which from the above proof is sufficient for concluding that $\rho\left(\prod_{i=1}^{P}\left(I-\mu I_{i} R\right)\right)<1$. Therefore, S-LMS also converges in the mean.

\section{ANALYSIS: CYCLO-STATIONARY SIGNALS}

Next, we consider the case when $\left\{x_{k}\right\}$ is cyclo-stationary. We limit our attention to S-LMS with alternate even and odd updates as shown in Figure 1. Let $\left\{x_{k}\right\}$ be a cyclo-stationary signal with period $L$. i.e, $R_{i+L}=R_{i}$. For simplicity, we will assume $L$ is even. For the regular LMS algorithm we have the following $L$ update equations

$$
E\left[V_{k+L}\right]=\prod_{i=0}^{L-1}\left(I-\mu R_{i+d}\right) E\left[V_{k}\right]
$$

for $d=1,2, \ldots, L$, in which case we would obtain the following sufficient condition for convergence

$$
0<\mu<\min _{i}\left\{2 / \lambda_{i, \max }\right\}
$$

where $\lambda_{i, \max }$ is the largest eigenvalue of the matrix $R_{i}$.

Define $A_{k}=\left(I-\mu I_{1} R_{k}\right)$ and $B_{k}=\left(I-\mu I_{2} R_{k}\right)$ then for the partial update algorithm the $2 L$ valid update equations are

$$
E\left[V_{k+L}\right]=\left(\prod_{i=0}^{\frac{L-1}{2}} B_{2 * i+1+d} A_{2 * i+d}\right) E\left[V_{k}\right]
$$

for $d=1,2, \ldots, L$ and

$$
E\left[V_{k+L}\right]=\left(\prod_{i=0}^{\frac{L-1}{2}} A_{2 * i+1+d} B_{2 * i+d}\right) E\left[V_{k}\right]
$$

for $d=1,2, \ldots, L$.

Let $\|A\|$ denote the spectral norm $\lambda_{\max }\left(A A^{H}\right)$ of the matrix $A$. Then for ensuring the convergence of the iteration (12) and (13) a sufficient condition is

$$
\left\|B_{i+1} A_{i}\right\|<1 \text { and }\left\|\dot{A}_{i+1} B_{i}\right\|<1 \text { for } i=1,2, \ldots, L
$$

Since we can write $B_{i+1} A_{i}$ as

$B_{i+1} A_{i}=\left(I-\mu R_{i}\right)+\mu I_{2}\left(R_{i}-R_{i+1}\right)+\mu^{2} I_{2} R_{i+1} I_{1} R_{i}$

and $A_{i+1} B_{i}$ as

$A_{i+1} B_{i}=\left(I-\mu R_{i}\right)+\mu I_{1}\left(R_{i}-R_{i+1}\right)+\mu^{2} I_{1} R_{i+1} I_{2} R_{i}$

we have the the following expression which upper bounds both $\left\|B_{i+1} A_{i}\right\|$ and $\left\|A_{i+1} B_{i}\right\|$

$$
\left\|I-\mu R_{i}\right\|+\mu\left\|R_{i+1}-R_{i}\right\|+\mu^{2}\left\|R_{i+1}\right\|\left\|R_{i}\right\|
$$

This tells us that the sufficient condition to ensure convergence of both (12) and (13) is

$$
\left\|I-\mu R_{i}\right\|+\mu\left\|R_{i+1}-R_{i}\right\|+\mu^{2}\left\|R_{i+1}\right\|\left\|R_{i}\right\|<1
$$

for $i=1, \ldots, L$

If we make the assumption that

$$
\mu<\min _{i}\left\{\frac{2}{\lambda_{i, \max }+\lambda_{i, \min }}\right\}
$$

and

$$
\delta_{i}=\left\|R_{i+1}-R_{i}\right\|<\max \left\{\lambda_{i, \min }, \lambda_{i+1, \min }\right\}=\eta_{i}
$$

for $i=1,2, \ldots, L$ then (18) translates to

$$
1-\mu \eta_{i}+\mu \delta_{i}+\mu^{2} \lambda_{i, \max } \lambda_{i+1, \max }<1
$$

which gives

$$
0<\mu<\min _{i=1}^{L}\left\{\frac{\eta_{i}-\delta_{i}}{\lambda_{i, \max } \lambda_{i+1, \max }}\right\}
$$

(20) is the sufficient condition for the convergence of S-LMS.

\section{EXAMPLE}

The usefulness of the bound on step-size for the cyclo-stationary case can be gauged from the following example. Consider a 2-tap filter and a cyclo-stationary $\left\{x_{k}\right\}$ with period 2 having the following auto-correlation matrices

$$
\begin{aligned}
& R_{1}=\left[\begin{array}{cc}
5.1354 & -0.5733-0.6381 i \\
-0.5733+0.6381 i & 3.8022
\end{array}\right] \\
& R_{2}=\left[\begin{array}{cc}
3.8022 & 1.3533+0.3280 i \\
1.3533-0.3280 i & 5.1354
\end{array}\right]
\end{aligned}
$$

For this choice of $R_{1}$ and $R_{2}, \eta_{1}$ and $\eta_{2}$ turn out to be 3.38 and we have $\left\|R_{1}-R_{2}\right\|=2.5343<3.38$. Therefore, $R_{1}$ and $R_{2}$ satisfy the assumption made for analysis. Now, $\mu=0.33$ satisfies the condition for the regular LMS algorithm but, the eigenvalues of $B_{2} A_{1}$ for this value of $\mu$ have magnitudes 1.0481 and 0.4605 . Since one of the eigenvalues lies outside the unit circle (12) is unstable for this choice of $\mu$. On the other hand (20) gives $\mu=0.0254$. For this choice of $\mu$ the eigenvalues of $B_{2} A_{1}$ turn out to have magnitudes 0.8620 and 0.8773 . Hence (12) is stable.

We have plotted the evolution trajectory of the 2-tap filter with input signal satisfying the above properties. We chose $W_{\text {opt }}=$ [0.4 0.5] in Figures 2 and 3. For Figure $2 \mu$ was chosen according to be 0.33 and for Figure $3 \mu$ was chosen to be 0.0254 . For simulation purposes we set $d_{k}=W_{o p t}^{H} S_{k}+n_{k}$ where $S_{k}=\left[\begin{array}{ll}s_{k} & s_{k-1}\end{array}\right]^{T}$ is a vector composed of the cyclo-stationary process $\left\{s_{k}\right\}$ with correlation matrices given as above, and $\left\{n_{k}\right\}$ is a white sequence, with variance equal to 0.01 , independent of $\left\{s_{k}\right\}$. We set $\left\{x_{k}\right\}=$ $\left\{s_{k}\right\}+\left\{v_{k}\right\}$ where $\left\{v_{k}\right\}$ is a white sequence, with variance equal to 0.01 , independent of $\left\{s_{k}\right\}$. 